\title{
Correction to: Vena cava filters in patients presenting with major bleeding during anticoagulation for venous thromboembolism
}

\author{
Meritxell Mellado ${ }^{1}$ Javier Trujillo-Santos ${ }^{2} \cdot$ Behnood Bikdeli $^{3,4,5} \cdot$ David Jiménez $^{6} \cdot$ Manuel Jesús Núñez $^{7}$. \\ Martin Ellis ${ }^{8}$. Pablo Javier Marchena ${ }^{9}$. Jerónimo Ramón Vela ${ }^{10}$. Albert Clara ${ }^{11}$. Farès Moustafa ${ }^{12}$.

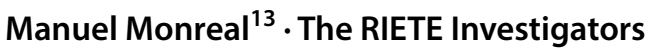

Published online: 20 June 2019

C Società Italiana di Medicina Interna (SIMI) 2019

\section{Correction to: Internal and Emergency Medicine https://doi.org/10.1007/s11739-019-02077-5}

In the original publication, part of conflict of statement was incorrectly published as "Dr. Bikdeli reports that he was approached by lawyers on behalf of plaintiffs in litigation related to IVC filters". The correct statement should read as "Dr. Bikdeli reports that he is a consulting expert (on behalf of the plaintiff) for litigation related to a specific type of IVC filters".
Publisher's Note Springer Nature remains neutral with regard to jurisdictional claims in published maps and institutional affiliations.
The original article can be found online at https://doi.org/10.1007/ s11739-019-02077-5.

Manuel Monreal

mmonreal.germanstrias@gencat.cat

1 Department of Angiology and Vascular Surgery, Hospital del Mar, Universitat Autònoma de Barcelona, Barcelona, Spain

2 Department of Internal Medicine, Hospital General Universitario de Santa Lucía, Murcia, Universidad Católica de Murcia (UCAM), Murcia, Spain

3 Division of Cardiology, Department of Medicine, Columbia University Medical Center/New York-Presbyterian Hospital, New York, NY, USA

4 Yale/YNHH Center for Outcomes Research and Evaluation (CORE), New Haven, CT, USA

5 Cardiovascular Research Foundation (CRF), New York, NY, USA

6 Respiratory Department, Hospital Universitario Ramón Y Cajal, IRYCIS, Madrid, Spain

7 Department of Internal Medicine, Complejo Hospitalario de Pontevedra, Pontevedra, Spain
Department of Haematology, Meir Hospital, Kfar Saba, Israel

9 Department of Internal Medicine and Emergency, Parc Sanitari Sant Joan de Deu-Hospital General, Barcelona, Spain

10 Department of Internal Medicine, Hospital Universitario Miguel Servet, Zaragoza, Spain

11 Department of Angiology and Vascular Surgery, Hospital del Mar, Universidad Autónoma de Barcelona, Barcelona, Spain

12 Department of Emergency, Clermont-Ferrand University Hospital, Université Clermont Auvergne, Clermont-Ferrand, France

13 Department of Internal Medicine, Hospital Universitari Germans Trias I Pujol, Universidad Autónoma de Barcelona, Carretera del Canyet s.n., Badalona, 08916 Barcelona, Spain 